\title{
ANÁLISE DE REDES SOCIAIS DAS TEMÁTICAS: GESTÃO DA INFORMAÇÃO E DA INOVAÇÃO
}

\author{
SOCIAL NETWORK ANALYSIS OF THE THEMES: \\ INFORMATION MANAGEMENT AND INNOVATION \\ ANÁLISIS DE REDES SOCIALES DE LAS TEMÁTICAS: \\ GESTIÓN DE LA INFORMACIÓN Y DE LA INNOVACIÓN
}

'Ana Clara Cândido, ${ }^{1}$ Patrícia Soares da Silva Bertotti, ${ }^{1}$ William Barbosa Vianna

${ }^{1}$ Universidade Federal de Santa Catarina

\author{
Correspondência \\ 'Ana Clara Cândido \\ Universidade Federal de Santa Catarina \\ Florianópolis, SC \\ E-mail: acc.anaclara@gmail.com \\ ORCID: http://orcid.org/0000-0003-1897-3946
}

Submetido em: $26-06-2017$

Aceito em: 22-07-2017

Publicado: 08-08-2017

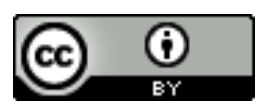

JITA: FJ. Knowledge management 
RESUMO: O objetivo do estudo é identificar relações entre os termos "gestão da informação" e "gestão da inovação" com vistas à formação de um portfólio bibliográfico para o estudo interdisciplinar do tema em Ciência da Informação. Trata-se de um estudo exploratório com aplicação de ferramenta de Análise de Redes Sociais na base de dados Science Direct entre 2010-2015 para construção de diagramas que representem a interação entre os conceitos mais frequentes da rede. Os resultados evidenciaram a baixa interação quantitativa entre os termos analisados e uma possível lacuna a ser explorada pela Ciência da Informação na sub-área da Gestão da Informação.

PALAVRAS ChAVE: Interdisciplinaridade. Gestão da informação. Inovação. Teoria do conceito. Organização da informação.

ABSTRACT: This study aims to identify relations between the terms "information management" and "innovation management" with a view to the formation of a bibliographic portfolio for the interdisciplinary study of the subject in Information Science. This is an exploratory study with application of Social Network Analysis tool in the Science Direct database between 2010-2015 to construct diagrams that represent the interaction between the most frequent concepts of the network. The results evidenced the low quantitative interaction between the terms analyzed and a possible gap to be explored by Information Science in the Information Management sub-area.

KEYWORDS: Interdisciplinary. Information management. Innovation. Concept theory. Information organization.

RESUMEN: El objetivo del estudio es identificar correlaciones entre los términos "gestión de la información" y "gestión de la innovación" con miras a la formación de una cartera bibliográfica para el estudio interdisciplinario del tema en Ciencia de la Información. Se trata de un estudio exploratorio con aplicación de herramienta de Análisis de Redes Sociales en la base de datos Science Direct entre 2010-2015 para la construcción de diagramas que representen la interacción entre los conceptos más frecuentes de la red. Los resultados evidenciaron la baja interacción cuantitativa entre los términos analizados y una posible laguna a ser explorada por la Ciencia de la Información en la sub-área de la Gestión de la Información.

PAlabras Clave: Interdisciplinariedad. Gestión de la información. Innovación. Teoría del concepto. Organizacíon de la informácion. 


\section{INTRODUÇÃ̃o}

Atualmente é consensual a importância da Gestão da Inovação no contexto de competitividade das empresas e soma-se a isso a importância do processo de gestão do conhecimento que é dependente de informação de qualidade (SORDI, 2008).

Entretanto, o termo "conhecimento", por vezes, é utilizado como sinônimo de "informação" e vice-versa. No campo da Ciência da Informação (CI), a redução da ambiguidade conceitual entre termo é essencial para o adequado desenvolvimento da pesquisa e das práticas na área e, no caso, do termo informação" ainda mais, pois integra o estatuto epistemológico de uma área cientifica.

Vick, Nagano e Santos (2009), explicam que "tanto a informação quanto o conhecimento são relacionais na medida em que dependem da transmissão de significados de forma dinâmica por intermédio das ações humanas".

Entretanto, a Gestão da Informação (GI), considerada uma sub-área da CI, segundo Davenport (1997), é um conjunto estruturado de atividades que espelha a forma pela qual uma organização captura, distribui e usa a informação. A acepção de Davenport concentra um consenso dentre os diversos entendimentos utilizados na CI.

Ao tratar a Gestão da Informação como um "conjunto de atividades", entende-se que seja um processo, ou seja, qualquer atividade isolada ou o conjunto destas que transformem recursos em produtos (entradas em saídas) de informação e possuam mecanismos de controle e verificação de qualidade e tal pode ser representado em seus elementos e etapas a fim de facilitar sua operacionalização. E tanto o conhecimento como a informação nascem a partir da criatividade humana, logo, é necessário que ocorra um enfoque na necessidade do 'usuário final', analisar o fator humano em todos os aspectos (DAVENPORT, 1998).

Segundo Fogl (1979, p.21-22), a informação compreende uma unidade de três elementos: a) Conhecimento (conteúdo da informação); b) Linguagem (um instrumento de expressão de itens de informação) e; c) Suporte (objetos materiais ou energia) e não há conexão direta entre informação e objeto, uma vez que a única fonte de origem da informação é o conhecimento, ou seja, a consciência humana.

Neste caso, uma aproximação entre os termos aponta para importância e centralidade do sujeito, ou mais claramente no caso de aplicação à processos de gestão, seja da inovação, da informação ou do conhecimento, da figura do decisor ou tomador de decisão no contexto organizacional.

Nesse sentido, um estudo que verifique ocorrências, incidências, eventuais correlações conceituais entre "gestão da informação" e "inovação" pode favorecer a identificação de onde

\begin{tabular}{l|l|}
$\mathrm{v} .15$ & $\mathrm{n} .3$ \\
\hline
\end{tabular}

p.670-692

set./dez. 2017


se situam os principais estudos e fomentar desenvolvimentos no campo da ciência da informação e, consequentemente colaborar com melhores práticas de gestão.

Dessa forma, pergunta-se: qual a incidência de uso do termo 'Gestão da Informação' nas publicações que abordam a gestão da inovação em diversas áreas do conhecimento?

Ao defender a ideia de que a Gestão da Informação pode oferecer uma relevante contribuição nos processos de inovação das organizações, o presente estudo tem como objetivo explorar a relação entre as temáticas 'gestão da informação e da inovação' em artigos publicados na base Science Direct, sendo o horizonte temporal das publicações de 2010 a 2015, divididos entre duas buscas nos anos de 2010 - 2013 e $2014-2015$.

O presente estudo é caracterizado como exploratório, tendo em vista que analisa duas temáticas já consolidadas separadamente, mas com escassos estudos sobre sua interrelação aplicada ao campo da Ciência da Informação.

Para tanto, foi utilizada a aplicação da Análise de Redes Sociais como ferramenta para identificar a relação entre as temáticas e, por meio dos softwares UCINET e Netdraw foram construídas matrizes de adjacência que permitem realizar uma análise e descrever algumas considerações relevantes da pergunta de pesquisa que se propõem responder.

O UCINET é um pacote de software para a análise de dados de redes sociais que inclui a ferramenta de visualização da rede NetDraw. O software possibilita uma análise das conexões e diferencia por cores os níveis das entidades envolvidas no estudo, permitindo uma melhor visualização e análise do mapeamento.

\section{GESTÃO DA INOVAÇÃO}

As publicações sobre o tema inovação tem ganhado espaço nos diversos campos do conhecimento ao longo dos últimos anos. No entanto, é possível observar diversos contextos onde o processo de inovação já está mais estabelecido, caracteristicamente em países onde a capacidade tecnológica é mais avançada e que apresentam maiores taxas de investimento em Pesquisa e Desenvolvimento (P\&D).

Esta situação coloca em voga a necessidade de amadurecimento de diversos aspectos para que o processo de inovação seja realizado de forma cada vez mais eficiente. Como é o caso da cultura de inovação, incentivos financeiros para o desenvolvimento inovador, recursos humanos, desburocratização, redes de parceiros, capacidade de absorção, processos de aprendizagem, acesso à informação, entre outros (CHOEN \& LEVINTHAL, 1989, 1990; DYER \& SINGH, 2003). 
Neste sentido, os resultados da gestão da inovação podem ser percebidos diretamente na competitividade empresarial e consequentemente refletir no crescimento sustentável. Além disto, a inovação possui um caráter de prospecção de oportunidades, busca de soluções para problemas percebidos, alterações no modelo de negócio convencional que possam agregar valor.

$\mathrm{O}$ potencial empreendedor e o contínuo investimento em $\mathrm{P} \& \mathrm{D}$ estão diretamente ligados aos processos de inovação. Tidd, Bessant e Pavitt (2008), mostram que a inovação não consiste apenas na geração de novas ideias, novos mercados, mas também em novas maneiras de agir nas empresas já estabelecidas no mercado.

Com relação à maneira como é realizada a inovação, Tidd, Bessant e Pavitt (2008, p. 107) indicam que é possível um entendimento onde "inovação é um processo, não um evento isolado, e precisa ser gerenciada como tal. As influências sobre esse processo podem ser manipuladas para afetar o resultado - ou seja, a inovação pode ser gerenciada".

A interação interna entre colaboradores e a interação com stakeholders externos é favorável ao desenvolvimento de inovações (CHESBROUGH, 2003). Por meio de troca de experiências entre os participantes do grupo e a compreensão do funcionamento da organização, torna-se possível o processo de aprendizagem e a inserção de rotinas capazes de maximizar os resultados pela gestão da inovação.

\subsection{Conceito de Inovação}

A Inovação é um processo contínuo que ocorre de forma ordenada e organizada, por isso, depende de um gerenciamento para que ocorra com plenitude.

O conceito de inovação é abordado pelo Manual de Oslo de forma ampla, conforme pode ser percebido na seguinte definição:

Uma inovação é a implementação de um produto (bem ou serviço) novo ou significativamente melhorado, ou um processo, ou um novo método de marketing, ou um novo método organizacional nas práticas de negócios, na organização do local de trabalho ou nas relações externas (FINEP, 2005).

Ainda na abordagem do FINEP (2005, p. 56) pode-se verificar um conceito mais voltado para os produtos e serviços, na qual "uma inovação pode ser mais estreitamente categorizada em virtude da implementação de um ou mais tipos de inovação, por exemplo, inovações de produto e de processo".

Para que um produto ou processo seja reconhecido como uma inovação, é preciso ser implementado e ter o seu valor reconhecido pelo mercado. De acordo com o FINEP (2005, p. 56), "uma empresa inovadora é aquela que implementou uma inovação durante o período de análise".

\begin{tabular}{l|l|} 
v.15 & n.3
\end{tabular}
p.670-692 set./dez. 2017 
Ainda sobre o conceito de inovação, torna-se importante mencionar que a inovação está diretamente relacionada à estratégia da organização, ao trabalho em equipe, à abertura e disposição para as mudanças. Para que isto seja possível acontecer, a organização precisa estar disposta e preparada para enfrentar os desafios e mudanças necessárias, nestes termos o apoio dos gestores é de fundamental importância.

\subsection{Tipos de Inovação segundo o Manual de Oslo}

Esta subseção baseia-se no principal documento de padronização dos conceitos relacionados à inovação em nível mundial, o FINEP (2005). As definições dos tipos de inovação estão ainda em desenvolvimento e estão menos estabelecidas do que as definições de inovação de produto e processo.

O FINEP (2005) aponta quatro formas de inovação: organização de produto, de processo, organizacional e de marketing. As inovações de produtos e processos com foco nos conceitos tecnológicos promovendo mudanças significativas nestes produtos e processos, potencializando desempenho e rendimento. As inovações organizacionais e de marketing se concentram em um conjunto mais abrangente de inovações, desde a implantação de novos métodos organizacionais às mudanças de design do produto e formas de elaboração do valor do produto ou serviço.

A contribuição primordial do economista Joseph Alois Schumpeter é citada no FINEP (2005) por ter tido grande influência nas teorias de inovação. Sua posição defende que por um processo dinâmico o desenvolvimento vai sendo conduzido, de maneira que as novas tecnologias vão eliminando as antigas, fenômeno descrito por ele como uma "destruição criadora".

Para Schumpeter (1934, apud FINEP, 2005, p.36) a inovação do tipo "radical" é mais complexa, exigindo maiores esforços e investimento, enquanto que inovações que visam "incrementar" darão continuidade, melhoria aos produtos e processos.

O autor propôs uma lista de cinco tipos de inovação:

i) introdução de novos produtos;

ii) introdução de novos métodos de produção;

iii) abertura de novos mercados;

iv) desenvolvimento de novas fontes provedoras de matérias-primas e outros insumos;

v) criação de novas estruturas de mercado em uma indústria.

O FINEP (2005) classifica a inovação em quatro áreas: produto, processo, marketing e organização. Entenda-se por produto, bens de consumo e serviços prestados. A inovação tecnológica de produto pode ser apresentada de duas maneiras: produtos tecnologicamente novos (onde o produto até poderá ter sido baseado em uma tecnologia anterior, mas com foco 
totalmente novo e diferenciado); produtos tecnologicamente aprimorados (produtos de uso simples que aprimorados melhoram seu desempenho significativamente, poderá envolver aprimoramento de custos também).

As definições de inovação de marketing e inovação organizacional não estão ainda bem definidas, estão em processo de desenvolvimento, ao contrário das definições de inovação de produto e de processo, sendo que as inovações de marketing se voltam para atender o mercado e consumidores com intuito de impulsionar as vendas e as inovações organizacionais estão relacionadas a mudanças que proporcionem um melhor desempenho da organização e seus negócios (FINEP, 2005).

O reconhecimento dessa identidade entre produto novo e aprimorado poderá gerar alguma dificuldade de reconhecimento em um primeiro momento, principalmente se tratando de setores que lidam com serviços prestados.

A busca por melhorias no desempenho, redução de custos e uma competitividade no mercado, são alguns dos fatores que impulsionam as empresas nesse caminho da inovação.

Nestes termos, o FINEP (2006, p. 37) aponta para a perspectiva schumpeteriana que "tende a enfatizar a inovação como experimentos de mercado e a procurar mudanças amplas e extensivas que reestruturam fundamentalmente indústrias e mercados".

Por fim, a inovação no atual contexto pode ser apresentada também como uma estratégia de negócio com o intuito de aumentar o desenvolvimento e o desempenho de um produto ou serviço. Isto ajuda a desmistificar a ideia de que inovação está diretamente relacionada estritamente à tecnologia, basta resgatar os tipos mencionados nesta seção para perceber o quão amplo pode ser este entendimento.

\subsection{Etapas do processo de Inovação}

No ambiente organizacional os efeitos esperados ocorrem de forma completamente diferente. Por isto muitos, diversas metodologias fornecem etapas e procedimentos para o estabelecimento dos passos do processo de inovação. Este procedimento para a inovação não deve ser considerado como padrão, pois em diferentes contextos e culturas fazem-se necessárias adaptações dos modelos e metodologias de acordo com a realidade da organização.

Stefanovitz e Nagano (2014, p. 463) defendem que "para se obter sucesso por meio da inovação, uma complexa gama de atividades deve acontecer de forma coordenada e sincronizada".

A Figura 1 apresenta uma proposta de consolidação de síntese dos processos de gestão da inovação e suas respectivas delimitações (STEFANOVITZ; NAGANO, 2014).

n.3

p.670-692




\begin{tabular}{|c|c|c|c|c|c|}
\hline & 1. Prospecção & 2. Ideação & $\begin{array}{l}\text { 3. Construção da } \\
\text { Estratégia }\end{array}$ & $\begin{array}{l}\text { 4. Mobilização de } \\
\text { Recursos }\end{array}$ & 5. Implementação \\
\hline 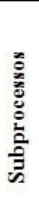 & $\begin{array}{l}\text { - Monitoramento de } \\
\text { tendências tecnológicas } \\
\text {. Monitoramento de } \\
\text { tendências de consumo } \\
\text {. Monitoramento de } \\
\text { competidores } \\
\text {. Construção de cenários }\end{array}$ & $\begin{array}{l}\text { Geração de novas ideias } \\
\text { Captação de ideias } \\
\text { Gestão de ideias }\end{array}$ & $\begin{array}{l}\text {. Construção do plano de } \\
\text { produtos } \\
\text {. Construção do roadmap de } \\
\text { tecnologias } \\
\text { - Gestão estratégica do portfólio } \\
\text { de projetos }\end{array}$ & $\begin{array}{l}\text {. Identificação de necessidades, } \\
\text { busca e mobilização de recursos } \\
\text {. Gestão operacional do } \\
\text { portfólio de projetos }\end{array}$ & $\begin{array}{l}\text { Processo de desenvolvimento } \\
\text { de tecnologias } \\
\text { Processo de desenvolvimento } \\
\text { de produtos }\end{array}$ \\
\hline$\frac{\operatorname{s}}{\partial}$ & $\begin{array}{c}\text { Sinais, tendências e } \\
\text { oportunidades do ambiente } \\
\text { geral e dos contextos } \\
\text { tecnológico e mercadológico }\end{array}$ & $\begin{array}{c}\text { Ideias, propostas e pré- } \\
\text { projetos que enderecem as } \\
\text { oportunidades identificadas }\end{array}$ & $\begin{array}{c}\text { Definição da direção } \\
\text { tecnológica e mercadológica, da } \\
\text { cadência do introduções e } \\
\text { seleção dos projetos a serem } \\
\text { executados }\end{array}$ & $\begin{array}{c}\text { Alocação de recursos, internos } \\
\text { ou externos, para a execução } \\
\text { dos projetos selecionados }\end{array}$ & $\begin{array}{l}\text { Introdução de produtos } \\
\text { inovadores no mercado }\end{array}$ \\
\hline & \multicolumn{5}{|c|}{ 6. Avaliação } \\
\hline 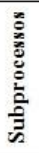 & \multicolumn{5}{|c|}{$\begin{array}{l}\text { Avaliação dos resultados e aprendizados dos projetos de inovação } \\
\text {. Avaliação de performance e melhoria continua do sistema de inovação }\end{array}$} \\
\hline 产 & \multicolumn{5}{|c|}{$\begin{array}{l}\text {. Incorporação de aprendizados obtidos ao longo da execução dos projetos } \\
\text {. Diagnóstico e monitoramento da performance dos processos de inovação para sua melhoria continua }\end{array}$} \\
\hline
\end{tabular}

Figura 1: Processos da Gestão da Inovação

Fonte: Stefanovitz e Nagano (2014, p.466)

A necessidade da coleta e análise de informações sobre um produto novo no mercado é um fator fundamental no processo de inovação. Esta ação ocorre na fase de Prospecção, onde o cliente final recebe uma nova oferta de produto ou serviço.

$\mathrm{Na}$ etapa Ideação se apresenta como fator extraordinário, novas propostas que apresentem ideias de novas soluções ou melhorias para algo já existente.

A etapa seguinte é a Construção da Estratégia, processo dividido em três etapas: análise, escolha e planejamento. A informação é fundamental neste processo e todas as coletas, sendo as análises realizadas nesta etapa fundamentais para a implementação da ideia (TIDD, BESSANT, PAVITT, 2008).

A Mobilização de Recursos se apresenta entre a construção da estratégia e a implementação, onde é necessário definir que recursos serão utilizados na execução.

A Implementação resulta de todas as fases anteriores e o resultado se apresenta em um produto ou serviço que está sendo aguardado por um mercado específico. O tempo e o custo são decisivos nesta fase, toda a organização se envolve nesta etapa. O processo de desenvolvimento de novos produtos e serviços são os dois principais processos que norteiam a implementações de inovações (STEFANOVITZ; NAGANO, 2014).

O processo de gestão da inovação (STEFANOVITZ; NAGANO, 2014) contempla também a etapa de Avaliação vai possibilitar que os processos de inovação das organizações melhorem constantemente, por meio de análises que apontem avanços e retrocessos, esse \begin{tabular}{|l|l|l|l|l|l}
\hline (C) RDBCI: Rev. Digit. Bibliotecon. Cienc. Inf. & Campinas, SP & v.15 & n.3 & p.670-692 & set./dez. 2017 \\
\hline
\end{tabular} 
processo de avaliação vai englobar duas fases: uma de revisão do projeto (logo após o lançamento) e outra de avaliação do sistema (possibilitando monitoramento).

Nas palavras de Stefanovitz e Nagano (2014, p. 466) "O desafio de inovar exige a criação de novas ideias, a superação de paradigmas tecnológicos e mercadológicos e um novo olhar sobre a realidade. Não se trata, pois, de um processo mecanicista com entradas e saídas padronizadas e repetitivas".

Portanto, reforça-se a ideia de que a ação de inovar não deve estar pautada apenas na modernização tecnológica, é preciso estabelecer uma cultura de inovação que envolva as pessoas e propicie bons insights de criatividade.

\section{GESTÃO DA INFORMAÇÃO}

A Gestão da Informação (GI), segundo Davenport (1998), é um conjunto estruturado de atividades que espelha a forma pela qual uma organização captura, distribui e usa a informação.

Ao tratar a Gestão da Informação como um "conjunto de atividades", entende-se que seja um processo, ou seja, qualquer atividade isolada ou o conjunto destas que transformem recursos em produtos (entradas em saídas) de informação e possuam mecanismos de controle e verificação de qualidade e tal pode ser representado em seus elementos e etapas a fim de facilitar sua operacionalização.

McGee \& Prusak (1994) sustentam que a informação não se limita aos dados coletados; mas se estende a dados coletados, organizados, ordenados, aos quais são atribuídos significados em determinados contextos.

Em um ambiente competitivo se torna fundamental a atuação da gestão da informação para estabelecer entre os colaboradores da organização um sentido de confiança e vínculo. Além disso, a manutenção desses atributos é de extrema importância para a imagem dessa organização. Quanto melhor for a gestão da informação na organização, melhor o desempenho da sua rede colaborativa, por exemplo.

De acordo com Choo (2003b, p. 19) “informação é o recurso que permite uma efetiva combinação e utilização dos outros fatores de produção - de facto, trata-se do meta-recurso que coordena a mobilização dos outros meios de forma a permitir o desempenho da organização".

Torna-se importante que cada organização encontre formas de administrar com eficiência seus processos de informação, com isso aumentando o aprendizado e a adaptação, transformando informação em conhecimento.

\begin{tabular}{l|l|} 
v.15 & n.3
\end{tabular}
p.670-692 set./dez. 2017 
Choo (2003b, p. 20), aborda que "como retórica, a gestão da informação é muitas vezes equiparada a gestão da tecnologia da informação ou à gestão de recursos de informação ou à gestão de políticas e modelos de informação", ideia que é apresentada na Figura 2.

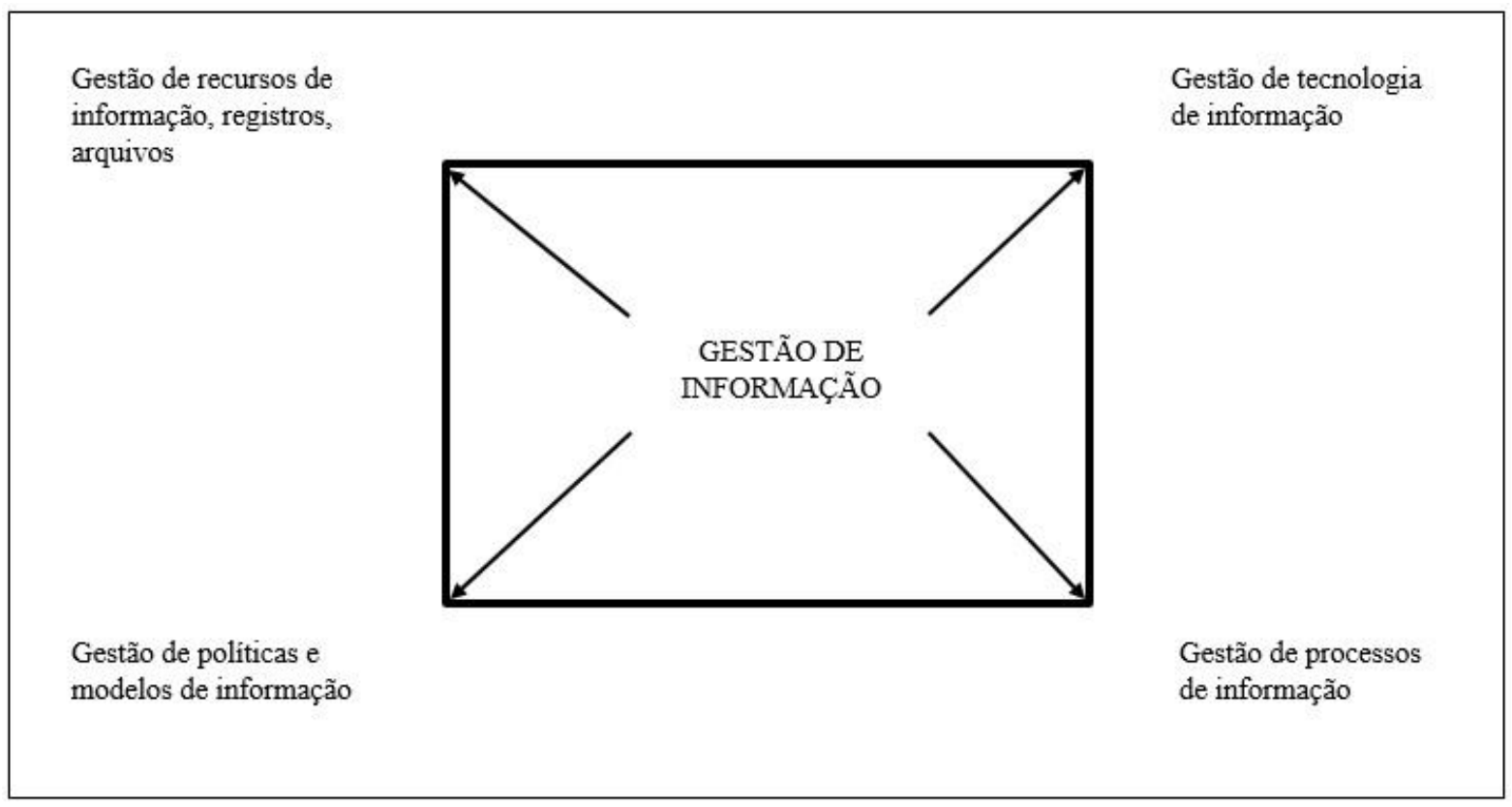

Figura 2: Gestão de informação

Fonte: Choo (2003b, p.19)

Por sua vez, a gestão da informação é descrita por Choo (2003, p. 19) como um "recurso estratégico da organização". Portanto, assume-se que gerir a informação significa buscar constantemente novas ideias, processos ou melhorias em produtos ou serviços. É no processo de gerenciar a informação e desenvolver o conhecimento que podem surgir os grupos inovadores, traçando metas, objetivos e trabalhando em equipe.

\subsection{O modelo informacional de Choo}

O modelo informacional de Choo (2003b) visa analisar as informações e o seu ciclo de atividades que estão interligadas, organizando o conhecimento:

O modelo processual descreve a gestão de informação como um ciclo contínuo de seis actividades estreitamente ligadas: identificação das necessidades de informação; aquisição de informação; organização e armazenamento de informação; desenvolvimento de produtos e serviços de informação; distribuição de informação; utilização de informação (CHOO, 2003b, p.58).

Na Figura 3 apresenta-se o modelo processual de gestão de informação e na sequência uma breve descrição deste processo. 


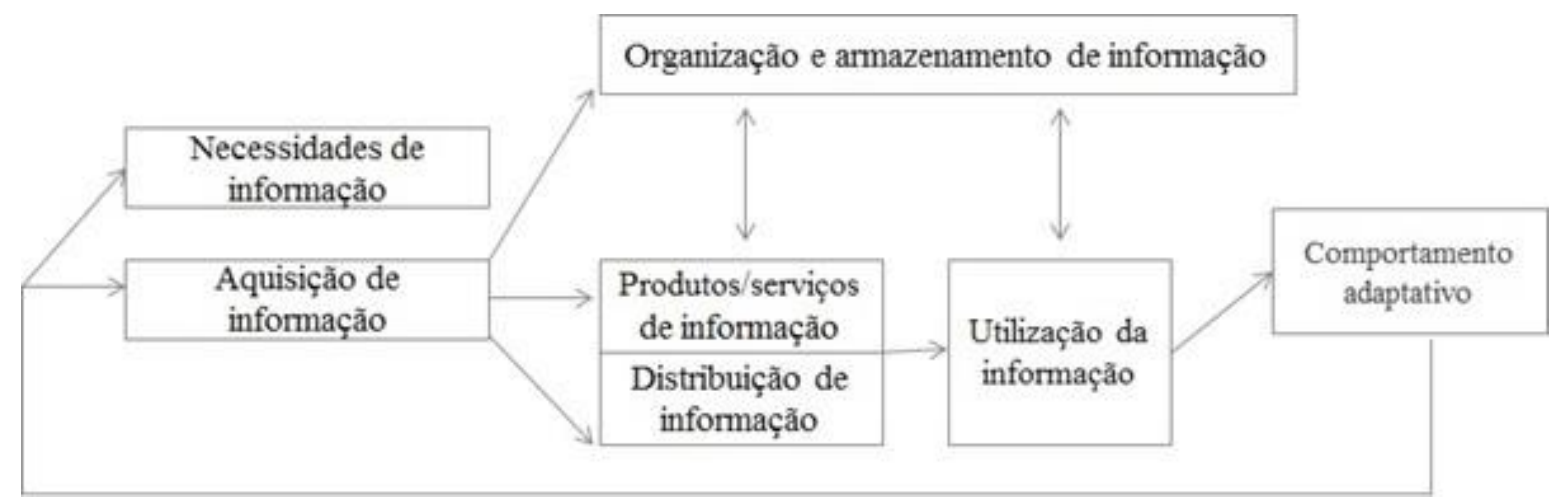

Figura 3. Ciclo da gestão de informação

Fonte: Choo (2003b, p.58)

Pode-se afirmar que, em diversas situações as organizações enfrentam adversidades na sua rotina, exigindo acesso à informação com precisão e rapidez na maioria dos casos. $\mathrm{O}$ foco destas necessidades deve aparecer em pontos apresentados pelos usuários e também em situações descritas por eles. Ou seja, a real necessidade de compreender o usuário e a necessidade informacional. Nas palavras de Choo (2003b, p.63) os requisitos de informação da organização são determinados a partir das necessidades de informação dos membros de círculos-chave que compõem a organização.

A necessidade de acessar e deter informações, bem como a adequação destas informações às demandas organizacionais proporciona e define complexas redes de planejamento a fim de adquiri-las (CHOO, 2003b). O acúmulo de informações gerado pela empresa demanda normas, procedimentos que auxiliem a gestão das necessidades informacionais encontradas em um primeiro momento. Da mesma forma, adequação e avaliação constantes destas fontes às suas necessidades.

Na organização e armazenagem, o objetivo principal é facilitar o acesso à informação. As organizações definem dentro de suas necessidades a melhor forma de armazenar e organizar o acesso às suas informações.

Os serviços e produtos de informação têm de acrescentar valor, realçando a qualidade da informação e melhorando a adequação entre a informação e as necessidades ou preferências dos utilizadores (CHOO, 2003b, p.59).

Na distribuição da informação o objetivo principal é uma distribuição eficiente da informação, contemplando sempre as necessidades do usuário e possibilitando o acesso com precisão e qualidade. A informação só passa a ser útil quando incute ao usuário um significado relevante, pois, a mesma informação poderá apresentar significados diferentes para diferentes usuários (CHOO, 2003a). 


\begin{abstract}
A utilização da informação destina-se a criação e aplicação de conhecimento através de processos de interpretação e de decisão. A utilização da informação na interpretação implica a construção social da realidade e a representação da informação e sua distribuição deveriam apoiar a interacção multinivelada do discurso social. A utilização da informação na tomada de decisão envolve a escolha de alternativas e o conteúdo e fornecimento de informação deviam encerrar em si a natureza cinética e não linear do processo de decisão (CHOO, 2003b, p. 59).
\end{abstract}

Portanto, a amplitude da informação vai estar diretamente relacionada ao usuário e a concepção ao uso que pretende fazer, seja na resolução de problemas ou na tomada de decisões.

\title{
4 PROCEDIMENTOS METODOLÓgICOS
}

Esta seção destina-se a apresentar os procedimentos metodológicos do estudo, tendo a finalidade de demonstrar e relatar as ferramentas, passos e procedimentos utilizados para o alcance dos resultados que serão posteriormente apresentados.

\subsection{Análise de Redes Sociais}

A relação entre as temáticas Gestão da Informação e da Inovação é avaliada no presente estudo através da aplicação da metodologia de Análise de Redes Sociais (ARS), tendo em vista a atender o objetivo deste estudo. A ARS utiliza um conjunto de medidas que permite o entendimento sobre diferentes padrões de interação entre indivíduos, instituições, e como no caso em questão a relação entre determinados conceitos teóricos.

A principal justificativa para o uso da ARS é a possibilidade de traçar um panorama sobre a relação das temáticas de gestão da informação e da inovação atendendo, desta forma, as expectativas do estudo exploratório que se propõe.

Nas palavras de Valk, Chapin e Gijsbers (2011, p. 26) "literature in the area of social network analysis (SNA) gives insight into concepts of network structure that may influence for instance the extent of diffusion of knowledge through a network".

\subsection{Coleta de dados e construção dos diagramas}

Para a coleta de dados, foram realizadas buscas por artigos publicados na base de dados science direct que continham em alguma parte do manuscrito os seguintes termos: 'information management' AND 'innovation management', desta forma para recuperar os artigos que continham de forma simultânea os termos.

A partir da identificação destes artigos, os dados foram organizados em forma de matrizes no excel de acordo com as suas palavras-chave. Após a coleta de artigo um a um, 
foram organizados os dados levantados, tendo o cuidado de eliminar palavras repetidas, termos no plural e singular, etc.

O horizonte temporal das publicações foi de 2010 a 2015, divididos entre os anos de 2010 - 2013 e 2014 - 2015. Portanto, a construção dos diagramas seguiu duas etapas de período conforme poderá ser observado nos resultados.

A partir da organização dos dados em matriz no excel, foram construídas matrizes de adjacência com base nas palavras chave de artigos publicados.

No primeiro período o total de artigos foi de 124, porém alguns não estavam disponíveis para consulta e assim foram consultados o total de 101 artigos.

No segundo período foram 133 artigos, porém disponíveis para consulta totalizaram 110 artigos.

Os softwares utilizados para a construção dos diagramas foram o UCINET e o NetDraw. Sendo que o primeiro passo (a coleta de dados) utilizou-se o excel.

\section{REDE DE RELAÇÃo ENTRE CONCEITOS: APLICAÇÃ̃o dA ANÁlISE DE REDES SOCIAIS}

Os conceitos de gestão da informação e gestão da inovação são assumidos no presente estudo como potenciais complementares para melhorar a performance competitiva das organizações e seu potencial interdisciplinar no campo da ciência da informação Além disso, ambos apresentam propostas de estruturação em aspectos considerados relevantes no atual contexto de constantes desenvolvimentos tecnológicos: informação e inovação.

Neste sentido, os resultados da aplicação da Análise de Redes Sociais (apresentados nas Figuras 4 e 5) evidenciam alguns conceitos de áreas distintas, mas na sua maioria no campo da gestão que estão relacionados aos estudos que abordam as temáticas em causa.

Um diagrama geral com todas as relações dos conceitos foi originado inicialmente, e devido a densidade da rede os dados foram tratados e dando origem à diagramas com os resultados mais significantes (maior número de relações existentes).

As Figuras 4 e 5 apresentam o resultado da interação de conceitos presente nos artigos publicados na base Science Direct com corte temporal nos períodos de 2010 - 2013 e 2014 2015, respectivamente. Em cada um dos diagramas é possível verificar a incidência de pequenas redes compostas entre conceitos. Este fato é explicado pelas diferentes áreas de aplicação dos artigos analisados. 
As redes representadas nos diagramas são compostas pelos nós (conceitos de origem) e pelos laços (relações entre os conceitos). Assim, os círculos representam os conceitos que dominam a força da interação e os quadrados representam conceitos que foram utilizados de forma conjunta no mesmo artigo publicado.

Embora tenham sido realizadas buscas em artigos que continham os dois conceitos simultaneamente em alguma parte do texto (information management AND innovation management), não houve incidência significativa dos termos e assim estes não estão evidenciados nos diagramas a seguir.

O Gráfico 1 apresenta os cinco journals que apresentaram o maior número de artigos baseado na busca realizada na base Science direct publicados entre 2010-2013.

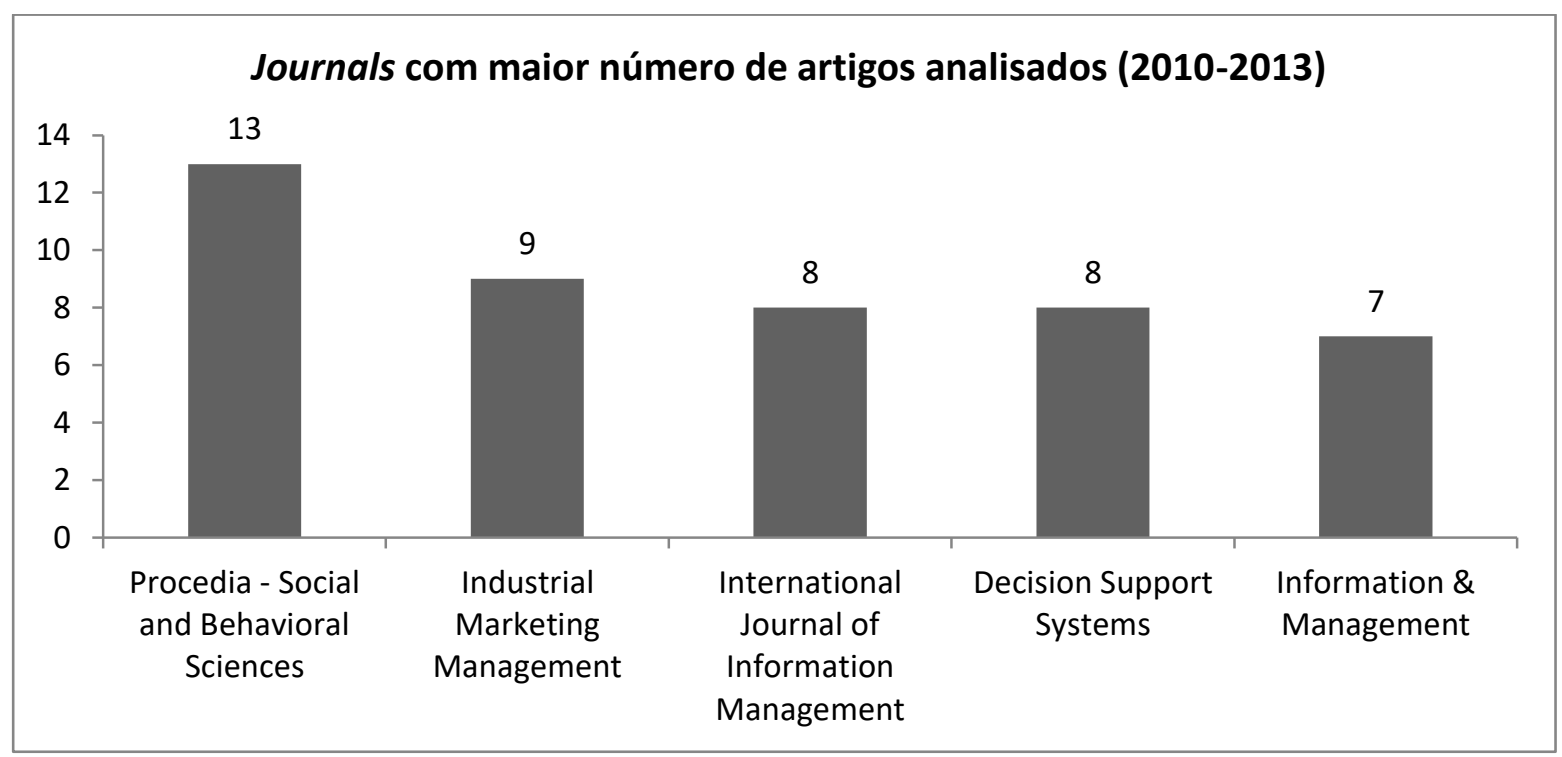

Gráfico 1 - Journals com maior número de artigos no período de 2010-1013

Fonte: Elaboração Própria

Os cinco journals com maior número de artigos representa quase $50 \%$ do total das publicações analisadas, sendo esta lista liderada pelo Periódico Procedia - Social and Behavioral Sciences. Este é um journal que recebe manuscritos de conferências que tenham acordo com a Elsevier (editora de conteúdos).

Destaca-se que periódico Procedia é de acesso aberto e recebe apenas manuscritos submetidos a conferências que tem um acordo com a Elsevier para publicação.

Considera-se que, para o avanço dos estudos de Gestão da Informação seja fundamental ampliar as consultas a bases de dados internacionais.

Kroeff et al (2015) identificou que não há muita novidade sendo publicada, em termos de conhecimento para a área, ou se, há novidade, as publicações recentes não estão sendo

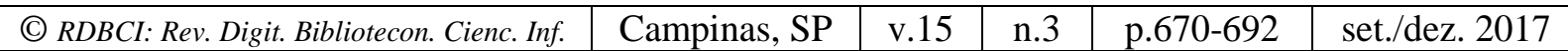


lidas e/ou citadas. $\mathrm{O}$ fato pode ser constatado, visto que os principais os autores citados nos artigos brasileiros analisados, se repetem constantemente, sendo que suas obras citadas não são novas (tendo em geral mais de dez anos de publicação).

É interessante mencionar que os demais journals apresentados no gráfico 1 estão relacionados à área de gestão, suporte à decisão e informação.

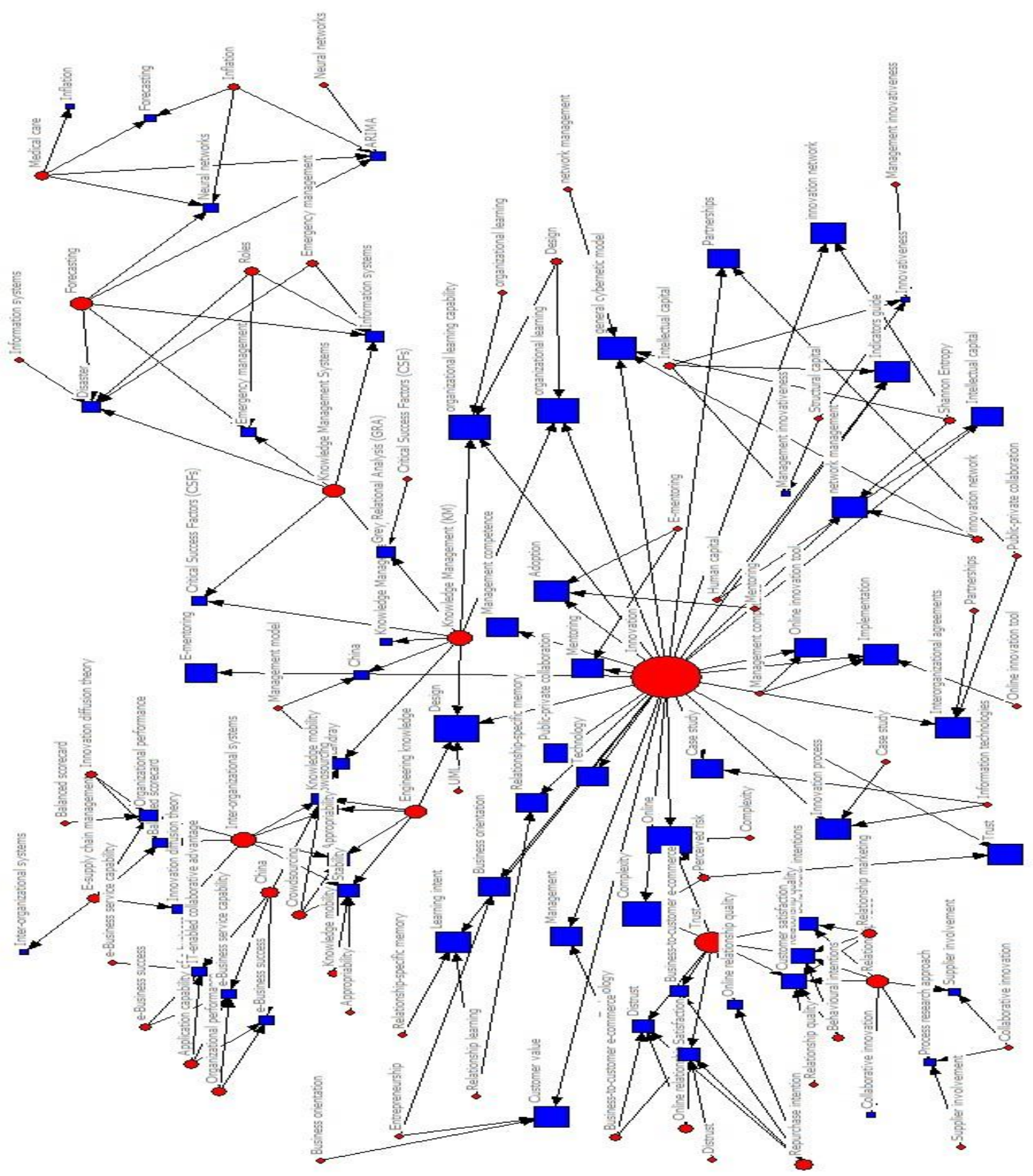

Figura 4 - Diagrama de termos Gestão da Informação e Gestão da Inovação (2010 - 2013) Fonte: Elaboração Própria

Algumas evidências são observadas a partir da análise dos diagramas. Os termos: inovação; confiança; sistemas interorganizacionais; gestão do conhecimento e sistemas de 
gestão do foram os que tiveram maior incidência nos artigos publicados no período de 2010 2013.

A seguir (Quadro 1) são demonstrados os termos mostraram relação mais frequente com os termos com maior incidência na rede:

Quadro 1 - Relações entre termos mais frequência nos artigos publicados entre 2010-2013

\begin{tabular}{|c|l|}
\hline $\begin{array}{c}\text { Termos com maior } \\
\text { incidência na rede }\end{array}$ & \multicolumn{1}{|c|}{ Termos com relações mais frequentes com os termos de maior } \\
incidência
\end{tabular}

Fonte: Elaboração Própria

Os resultados evidenciam a lacuna existente da gestão da informação em estudos sobre o processo de inovação. A análise dos termos que aparecem com frequência na rede mostra a transição do paradigma de gestão da inovação de forma colaborativa. Como é o caso de: gestão de redes; redes de inovação; acordos interorganizacionais; colaboração pública privada. No entanto, o recurso informacional não foi observado como fator predominante na rede composta dos termos presentes em artigos publicados entre 2010 e 2013. 


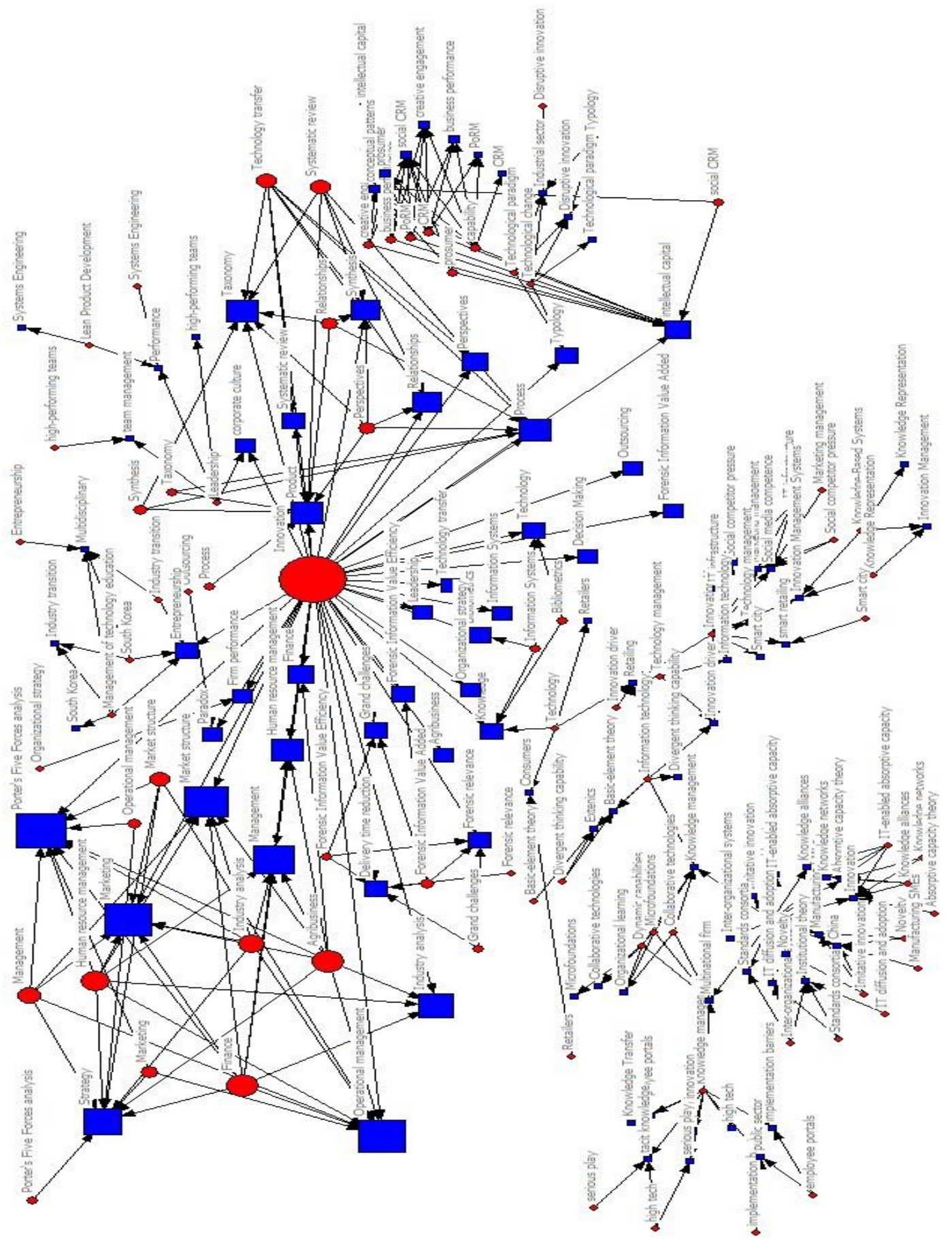

Figura 5 - Diagrama de termos Gestão da Informação e Gestão da Inovação (2014 - 2015) Fonte: Elaboração Própria

O diagrama sobre o período de 2014 - 2015 (Figura 5) apresenta-se com uma rede mais composta, com maior incidência de conceitos frequentes. Entre estes, os mais frequentes são: inovação, finanças, agronegócio, análise da indústria, gestão e gestão de recursos humanos. 
O Quadro 2 apresenta os termos que tiveram relação mais frequente e os termos com maior incidência na rede:

Quadro 2 - Relações entre termos mais frequência nos artigos publicados entre 2014-2015

\begin{tabular}{|c|c|}
\hline $\begin{array}{l}\text { Termos com maior } \\
\text { incidência na rede }\end{array}$ & $\begin{array}{c}\text { Termos com relações mais frequentes com os termos de maior } \\
\text { incidência }\end{array}$ \\
\hline Inovação & $\begin{array}{l}\text { Produto; processo; tipologia; taxonomia; cultura corporativa; } \\
\text { empreendedorismo; performance da firma; gestão de operações; finanças; } \\
\text { tempo de redução de entrega; relevância forense; grandes desafios; } \\
\text { estratégia organizacional; liderança; transferência de tecnologia; tomada de } \\
\text { decisão; outsourcing; valor acrescentado de informações forenses; } \\
\text { perspectivas; relacionamento; síntese; tecnologia }\end{array}$ \\
\hline Finanças & Marketing; análise das 5 forças de Porter; gestão; estrutura de mercado \\
\hline Agronegócio & $\begin{array}{l}\text { Gestão; estrutura de mercado; análise da indústria; análise das } 5 \text { forças de } \\
\text { Porter }\end{array}$ \\
\hline Análise da indústria & Marketing; gestão; análise das 5 forças de Porter \\
\hline Gestão & Estratégia; análise das 5 forças de Porter; finanças; marketing \\
\hline $\begin{array}{l}\text { Gestão de recursos } \\
\text { humanos }\end{array}$ & Marketing; análise das 5 forças de Porter \\
\hline
\end{tabular}

Fonte: Elaboração Própria

Por fim, nota-se que os termos predominantes são oriundos da área de gestão. Embora os conceitos utilizados para a coleta de dados não tenham apresentado frequência significativa e assim não encontram-se nos diagramas, vários conceitos inerentes aos processos de inovação e gestão da informação foram identificados nas relações da rede de conceitos: estratégia organizacional, sistemas de informação, tomada de decisão, capital intelectual, gestão da tecnologia, gestão do conhecimento.

A ausência do termo gestão da informação pode refletir a lacuna de estudos empíricos aproximados ao campo da inovação, mesmo embora a potencialidade da interação destes processos já tenho sido observada pela literatura.

Em contraste a esta situação, o termo 'gestão do conhecimento' é evidenciado em artigos no período de 2010-2013 com frequência de relação com os termos: design, aprendizado organizacional, fatores críticos de sucesso, Grey Relational Analysis (GRA).

Destaca-se que o termo 'gestão do conhecimento' e 'sistemas de gestão do conhecimento' estavam entre os mais frequentes no período analisado, conforme descrito anteriormente. 
A incidência no período de 2014-2015 do termo gestão do conhecimento apresenta relações com os seguintes termos: tecnologias colaborativas e aprendizagem organizacional.

A incidência no período de 2014-2015 do termo gestão do conhecimento apresentou relações com os seguintes termos: tecnologias colaborativas e aprendizagem organizacional.

A predominância de ocorrência do termo "gestão do conhecimento" nos permite afirmar que sua ocorrência tem sido quantitativamente maior como "palavra-chave" e indica a necessidade de outros estudos, de matriz qualitativa para analisar quais são os conceitos envolvidos e que ambiguidades estão, eventualmente envolvidas em seu uso.

O Gráfico 2 apresenta os journals mais frequentes nos artigos analisados no período de 2014- 2015, da mesma forma como se apresentou para o período anterior.

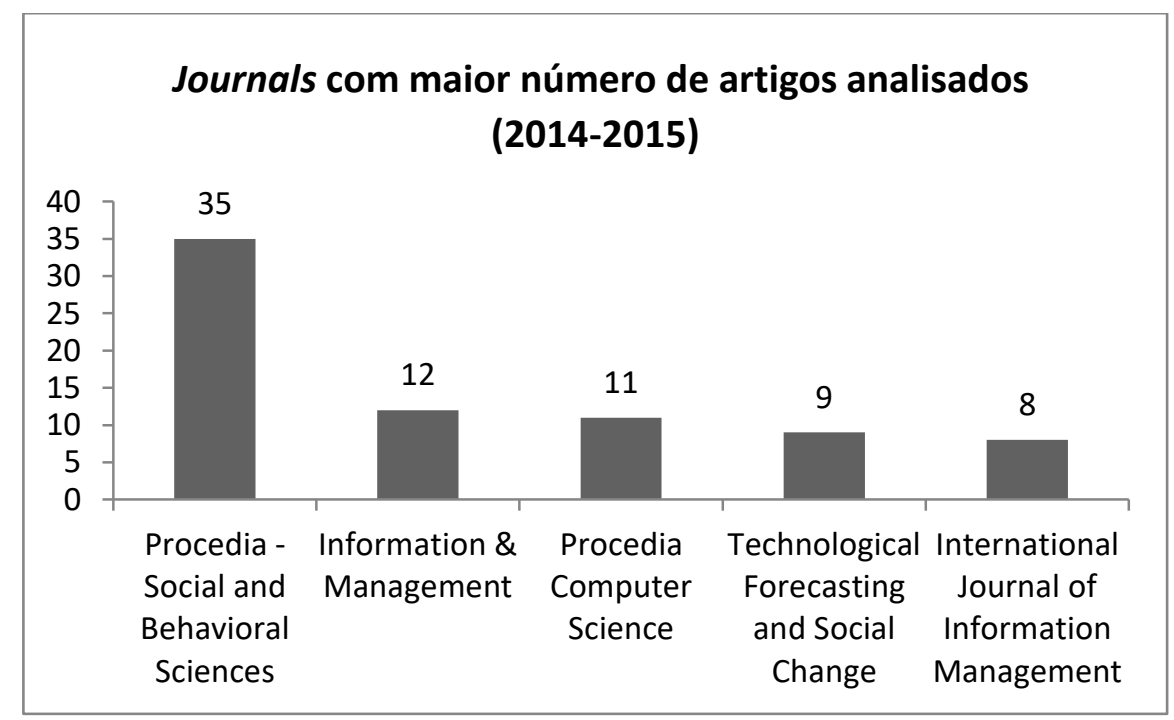

Gráfico 2 - Journals com maior número de artigos no período de 2014-1015

Fonte: Elaboração Própria

O journal 'Procedia - Social and Behavioral Sciences' continua liderando a lista, com um total de 35 artigos daqueles que continham em alguma parte do texto os termos 'information management' AND 'innovation management'. Conforme mencionado anteriormente, podendo ser justificado pelo seu escopo e por se tratar de manuscritos de conferências que possuam acordo com a Elsevier.

Em relação ao período anterior, mostrado anteriormente, novos periódicos aparecem na lista dos 5 mais frequentes, como é o caso de 'Procedia - Computer Science' e 'Technological Forecasting and Social Change'

A tabela 1 a seguir apresenta algumas informações sobre os diagramas apresentados anteriormente para os períodos de 2010-2013 e 2014-2015. 
Tabela 1 - Informação sobre os diagramas de termos Gestão da Informação e da Inovação

\begin{tabular}{|l|c|c|}
\hline \multicolumn{1}{|c|}{ Indicador } & Período 2010-2013 & Período 2014-2015 \\
\hline Número de laços & 176 & 295 \\
\hline Número de componentes & 828 & 994 \\
\hline Centralidade de grau & $3,4 \%$ & $3,6 \%$ \\
\hline
\end{tabular}

Fonte: Elaboração Própria

A análise da centralidade permite compreender o posicionamento dos diferentes atores na rede, neste caso as palavras-chave. A centralidade de grau (degree centrality), que expressa o número de laços diretos que um ator (palavra-chave) tem com os restantes atores (palavras-chave) da rede, sendo uma medida do grau de atividade.

O diagrama do período de 2010 - 2013 é formado por 828 componentes, apresentando 176 laços de relações. O grau de centralidade desta rede foi de $3,4 \%$ significando baixo número de palavras-chave predominantes nas relações com as demais palavras-chave.

Já no período de 2014 - 2015, o número de componentes é de 994 com 295 laços de relações. Em comparação com o período anterior, o aumento do número de laços foi mais acentuado do que o número de componentes, embora este último também tenha sido maior. $\mathrm{O}$ grau de centralidade não apresentou alterações representativas, sendo o seu valor de 3,6\% permanecendo a característica de uma rede formada por poucas palavras-chave predominantes.

\section{CONSIDERAÇÕES FINAIS}

O presente estudo objetivou explorar a relação entre as temáticas gestão da informação e da inovação em artigos publicados na base Science Direct, sendo o horizonte temporal das publicações de 2010 a 2015, divididos entre duas buscas nos anos de 2010 - 2013 e 2014 2015.

O uso do termo 'gestão do conhecimento' foi destacado nas redes representadas pelos diagramas, conforme mencionado na seção dos resultados. Nesse caso, os resultados corroboraram a afirmação de Donate e Pablo (2014, p. 360) ao analisarem o papel da liderança orientada para o conhecimento em práticas de gestão do conhecimento e inovação "Emerging in the nineties, knowledge management (KM) is a well established discipline in the academic field and business world alike" onde os autores seguem afirmando que a importância e o uso da gestão do conhecimento são inquestionáveis.

Por outro lado, os resultados evidenciaram uma baixa ocorrência para o termo "gestão da informação" nos artigos identificados em associação booleana com o termo "gestão da

\begin{tabular}{|c|c|c|c|c|c|}
\hline (C) RDBCI: Rev. Digit. Bibliotecon. Cienc. Inf. & Campinas, SP & v.15 & n. 3 & p.670-692 & set./dez. 2017 \\
\hline
\end{tabular}


inovação". Considerando a possibilidade de que ambiguidades conceituais possam envolver o uso como sinônimos dos termos "informação" e "conhecimento" e que, para o campo da ciência da informação, a redução da ambiguidade é fundamental para o adequado uso teórico e prático dos conceitos em comunidades de prática, considera-se necessário um aprofundamento qualitativo do uso dos termos na massa bibliográfica resgatada.

A Gestão da Informação é um "recurso estratégico da organização". fundamental para a tomada de decisão no desenvolvimento de uma determinada inovação nas etapas: prospecção, ideação, construção da estratégia, mobilização dos recursos e implementação (conforme Figura 1).

$\mathrm{Na}$ exposição dos diagramas verificou-se que parece existir uma lacuna em estudos que tratem da gestão da informação no âmbito de processos de inovação. Esta situação é evidenciada pela ausência de relação entre estes conceitos nos estudos publicados na base Science Direct, conforme tratado na aplicação da Análise de Redes Sociais.

Os diagramas apresentados no estudo permitiram uma compreensão sobre quais temas estão sendo mais frequentemente tratados conjuntamente nas publicações recentes em termos quantitativos de ocorrência de palavras-chave.

Este foi um exercício que poderá ser replicado em estudos futuros utilizando outras bases (nacionais e internacionais), para área da Ciência da Informação e áreas correlatas com o intuito de verificar a incidência de publicações que integrem estes conceitos.

E mais do que isso, um aprofundamento da análise dos resultados encontrados em termos qualitativos quanto aos conceitos declarados ou implícitos, poderá retornar resultados que associem mais fortemente ao que se tem considerado como gestão da informação no campo da ciência da informação ou confirmar a escassez de abordagens que correlacionem o termo com "gestão da inovação", o que se considerou uma lacuna a ser explorada em estudos futuros no campo da CI.

\section{REFERÊNCIAS}

CHESBROUGH, Henry. Open Innovation: The New Imperative for Creating and Profiting from Technology. Boston: Harvard Business School Press, 2003.

CHOO, Chun Wei. A organização do conhecimento: como as organizações usam a informação para criar significado, construir conhecimento e tomar decisões. São Paulo: Senac, 2003a. 425 p.

CHOO, Chun Wei. Gestão de Informação para a Organização Inteligente: A arte de explorar o meio ambiente. Lisboa: Caminho, 2003b. 365 p. 
DAVENPORT, Thomas H. Ecologia da Informação: Por que só a tecnologia não basta para o sucesso na era da informação. São Paulo: Futura, 1998. Tradução: Bernadette Siqueira Abrão. Disponível em: < http://amormino.com.br/livros/20141114-ecologia-informacao.pdf $>$. Acesso em: 19 jun. 2017.

DONATE, Mario J; PABLO, Jesús D.S. The role of knowledge-oriented leadership in knowledge managament practices and innovation. Journal of Business Research, v. 68, pp. 360-370, 2014.

DYER, Jeffrey H; SINGH, Harbir. Utilizando alianças para construir vantagem competitiva em tecnologias emergentes. In: Day, G.S., Schoemaker, P.J.H., Gunther, R.E. Gestão de Tecnologias Emergentes: a visão de Wharton School. Porto Alegre: Bookman, 2003, pp. 312- 327.

FINEP. Manual de Oslo. 2005. Disponível em: < http://www.finep.gov.br/images/apoio-efinanciamento/manualoslo.pdf >. Acesso em: 15 maio 2017.

FOGL, J. Relations of the concepts 'information' and 'knowledge'. International Fórum on Information and Documentation, The Hague, v.4, n.1, p. 21-24, 1979.

KROEFF, Marcia Silveira et al. Análise de citações dos artigos publicados em periódicos da área da Ciência da Informação que versam sobre gestão da informação.. RBBD. Revista Brasileira de Biblioteconomia e Documentação, São Paulo, v. 11, n. 1, p. 41-65, jul. 2015.

MCGEE, J. V.; PRUSAK, L. Gerenciamento estratégico da informação: aumente a competitividade e a eficiência de sua empresa utilizando a informação como uma ferramenta estratégica. Rio de Janeiro: Campus, 1994.

SORDI, José Osvaldo de. Administração da Informação: FUNDAMENTOS E PRÁTICAS PARA UMA NOVA GESTÃO DO CONHECIMENTO. São Paulo: Saraiva, 2008. 185 p.

STEFANOVITZ, Juliano Pavanelli; NAGANO, Marcelo Seido. Gestão da inovação de produto: proposição de um modelo integrado. Production, [s.1.], v. 24, n. 2, p.462-476, jun. 2014. FapUNIFESP (SciELO). http://dx.doi.org/10.1590/s0103-65132013005000062.

TIDD, Joe; BESSANT, John; PAVITT, Keith. Gestão da Inovação. 3. Ed. Porto Alegre: Bookman, 2008. 600 p.

VALK, Tessa van der; CHAPPIN, Maryse M.H; GIJSBERS, Govert W. Evaluating innovation networks in emerging technologies. Technological Forecasting \& Social Change, v. 78, p. 25-39, 2011.

VICK, Thais; NAGANO, Marcelo Seido; SANTOS, Fernando César Almada. Aportes da gestão da informação para a criação de conhecimento em equipes de inovação. Perspectivas em Ciência da Informação, Belo Horizonte, v. 14, n. 2, p.204-219, maio 2009. 

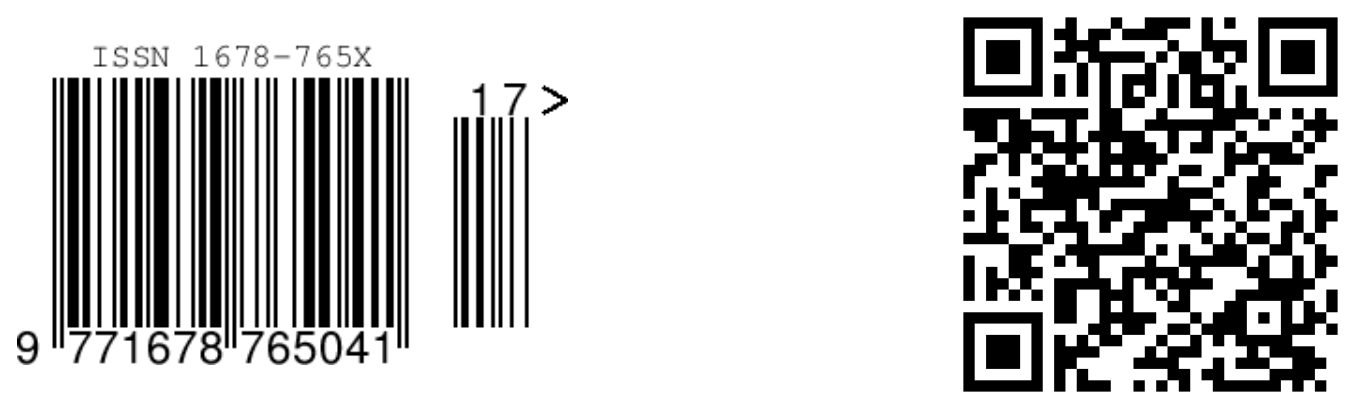